\title{
Oral Supplementation of Tocotrienol-Rich Fraction Alleviates Severity of Ulcerative Colitis in Mice
}

\author{
Tzuen Yih SAW ${ }^{1,2}$, Najib Abdul MALIK ${ }^{1,2}$, Kee Pah LIM ${ }^{1,2}$, Cheryl Wei Ling Teo ${ }^{1,2}$, \\ Esther Sook Miin WoNG ${ }^{3}$, San Choon $\mathrm{KONG}^{4}$, Chee Wai FoNG ${ }^{1,2}$, \\ Jordan PETKOV ${ }^{2}$ and Wei Ney YAP ${ }^{1,2, *}$ \\ ${ }^{1}$ Research \& Development Department, Davos Life Science, Singapore \\ ${ }^{2}$ Research \& Development Department, KL-Kepong Oleomas Sdn Bhd (KLK Oleo), Malaysia \\ ${ }^{3}$ Institute of Medical Biology, Agency of Science Technology and Research (A*STAR), Singapore \\ ${ }^{4}$ Gastroenterology \& Hepatology Department, Singapore General Hospital, Singapore
}

(Received February 21, 2019)

\begin{abstract}
Summary Ulcerative colitis (UC) is characterized by damaged colonic mucosa and submucosa layers that are caused by excessive inflammatory reactions and oxidative stress. This study aimed to examine the use of tocotrienol-rich fraction (TRF) in mitigating damages caused by UC on the colon epithelium. Dextran sulfate sodium (DSS)-induced UC mice were treated with vehicle control, TRF, alpha-tocopherol $(\alpha \mathrm{TP})$ and 5-aminosalicylic acid (5-ASA). Observable clinical signs, quality of stool, histopathological scoring, inflammatory and oxidative markers were assessed. Vitamin E levels of colons and plasma were quantified. Oral supplementation of TRF significantly reduced the severity of DSS-induced UC by lowering the disease activity index (DAI) and histopathological inflammatory scoring. TRF also attenuated the DSS-induced enlargement of spleen and shortening of the colon. TRF has demonstrated marked anti-inflammatory and antioxidative properties indicated by the attenuation of DSS-induced upregulation of inflammation and oxidative stress markers including interleukin (IL)-6, IL-17, tumor necrosis factor (TNF)- $\alpha$, myeloperoxidase (MPO), cyclooxygenase-2 (COX-2), nitric oxide (NO), malondialdehyde (MDA) and pNF- $\kappa$ B. These improvements were similar to that of 5-aminosalicylic acid (5-ASA) treatment. In contrast, $\alpha \mathrm{TP}$ did not demonstrate evident clinical and histopathological improvements. The superior protective effect of TRF may be ascribed to the preferential absorption of TRF by the gut mucosa. TRF alleviated the signs and symptoms of acute UC in murine model via the reduction of local inflammatory reactions and oxidative stress. These effects suggested that TRF could serve as a gut health supplement for preventive measures for UC condition in patients. Key Words vitamin E, inflammatory bowel disease, anti-inflammatory, anti-oxidative, 5-aminosalicylic acid
\end{abstract}

Intestinal inflammation, including inflammatory bowel disease (IBD) describes clinical conditions with uncontrolled or recurring immune responses and severe inflammation of the gastrointestinal tract (1). The two most common IBD are ulcerative colitis (UC) and Crohn's disease (CD). The former one causes continuing inflammation in certain part of the digestive tract mainly in colon while the latter one causes inflammation at any places along the lining of the digestive tract (2). Although the etiology of IBD remains poorly understood, it is postulated that the onset of the disease

\footnotetext{
*To whom correspondence should be addressed.

E-mail: weiney.yap@davoslife.com

Abbreviations: 5-ASA, 5-aminosalicylic acid; CD, Crohn's disease; COX-2, cyclooxygenase-2; DAI, disease activity index; DSS, dextran sulfate sodium; IBD, inflammatory bowel disease; IL, interleukin; MDA, malondialdehyde; MPO, myeloperoxidase; NF- $\kappa \mathrm{B}$, nuclear factor kappa-light-chain-enhancer of activated B cells; NO, nitric oxide; T3, tocotrienol; TNF- $\alpha$, tumor necrosis factor alpha; TP, tocopherol; TRF, tocotrienolrich fraction; UC, ulcerative colitis.
}

is triggered by multifactorial insults, such as microbes, toxins, pollutants, loss of defective epithelial barrier and genetic mutation (3). These insults lead to exaggerated inflammatory responses that damage mucosa and submucosa layers in the colon and rectum epithelium. These inflammatory responses trigger an infiltration of the lamina propria by both innate (neutrophils, macrophages, dendritic cells and natural killer T cells) and adaptive (B and T cells) immune cells. Increased activation and recruitment of these cells enhance local levels of tumor necrosis factor (TNF)- $\alpha$ and induce expression of several proinflammatory interleukins (IL) including IL-1, IL-6, IL-1 $\beta$, and cytokines of the IL-23-Th1 7 pathway (4). In most cases, ulcers form uniformly along the entire colon of patients contributing the epithelium to be reddish, swollen and bleeding. IBD patients will also be affected by various of symptoms including diarrhea, constipation, faecal blood, weight loss, loss of appetite, fatigue, and joint pain which can last for a few days to a few weeks. During periods of remission, no symptoms of the disease are noticeable yet the relapse at varying 
intensity can occur at any time throughout the patient's life.

Current medications aim to suppress or modulate intestinal inflammation by interfering with multiple stages of inflammatory cascade when relapse occurs (5). Standard-of-care treatment includes 5-aminosalicylic acid (5-ASA, or mesalamine) for mild to moderate symptoms and prednisolone for moderate to severe symptoms (6). Other drugs include azathioprine, 6-mercaptopurine, methotrexate, cyclosporine, and TNF- $\alpha$ blocking agents (7). While the efficacy of these IBD drugs are only successful in about two-thirds of patients (8), they are associated with undesirable adverse effects including nausea, cramping, flatulence, pancreatitis, abdominal pain, headache and anaphylaxis (9). Therefore, a surge of interest in studying natural products with potent anti-oxidative and anti-inflammatory properties has emerged so that remission can be maintained for a longer period and relapses can be adjourned as soon as possible, with minimal adverse effects.

Vitamin E is a group of phytochemicals including tocopherols (TPs) and tocotrienols (T3s) that are naturally derived from plant sources such as palm, rice bran and annatto. Vitamin E is the major lipophilic antioxidant in cellular membrane to provide significant source of antioxidant activities which protects membrane lipids from lipid peroxidation by scavenging chain-carrying lipid peroxyl radicals. Depending on the location and number of methyl groups on the chromanol ring, T3s and TPs are classified into four isoforms: alpha $(\alpha)$, beta $(\beta)$, gamma $(\gamma)$ and delta $(\delta)$. Numerous scientific evidences have demonstrated T3 and TP differ in their biological responses. T3s possess stronger anti-oxidative and anti-inflammatory properties than $\alpha \mathrm{TP}$ (a more common form of vitamin E) because T3s have three double-bonds in the farnesyl chain that cause greater planar distortion and disruption when incorporated into plasma membrane and cytoplasm (10). T3s have been associated with lowering of proinflammatory mediators such as IL-6, TNF- $\alpha$, IL-1 $\beta$, cyclooxygenase-2 (COX-2), prostaglandin E2 (PGE2), as well as oxidative molecules such as reactive oxygen species (ROS) and reactive nitrogen species (RNS) in cell line studies $(11,12)$.

Thus, given the established studies suggesting antioxidative and anti-inflammatory properties of $\mathrm{T} 3$, the work presented here aimed to examine if supplementation of tocotrienol-rich fraction (TRF) can reduce the inflammatory reactions and oxidative stress in the colon to improve the severity of UC. Alpha tocopherol ( $\alpha \mathrm{TP})$, a well-known form of vitamin E, was used as a comparator. To the best of our knowledge, this is the first report to compare the efficacy of TRF and $\alpha \mathrm{TP}$ in alleviating DSS-induced UC in mice model. This study further deciphered the mechanism responsible for the superior gut protective effects of TRF, in comparison to $\alpha \mathrm{TP}$.

\section{MATERIALS AND METHODS}

Materials. Tocotrienol-rich fraction (DavosLife E3) was supplied by Davos Life Science Sdn Bhd (Malysia) with the composition consisted of $70 \%$ T3 $(27 \% \alpha \mathrm{T} 3$,
$3 \% \beta \mathrm{T} 3,32 \% \gamma \mathrm{T} 3,8 \% \delta \mathrm{T} 3)$ and $24.5 \% \alpha \mathrm{TP}$. High purity of $\alpha \mathrm{TP}(94 \%)$ and 5-aminosalicylic acid (5-ASA) were purchased from Sigma-Aldrich (St. Louis, MO). The dextran sulfate sodium (DSS, 36-50 kDa) was bought from MP Biochemicals (Irvine, CA).

Experimental animals. All animals received care in compliance with National Advisory Committee on Laboratory Animal Research guidelines. Experimental protocols were reviewed and approved by the Institutional Animal Care and Use Committee (IACUC number: 151087) at Biological Resource Centre (BRC), Agency of Science Technology and Research (A*STAR), Singapore. Male C57BL/ 6 mice, aged 6 to $8 \mathrm{wk}$, were obtained from InVivos (Singapore). They had ad libitum access to water and a standard diet with no added vitamin E (Research Diet Inc., New Brunswick, NJ) to minimize the inference of $\alpha$-tocopheryl acetate from the diet to the supplementation groups. They were maintained on a 12-h light/dark cycles in specific pathogen-free facilities under standard environmental condition at BRC. Upon arrival, the mice were acclimatized for $1 \mathrm{wk}$ before the experiment to ensure the animals were settled into the environment.

Experiment design. The DSS method has been established to induce inflammation-driven $\mathrm{UC}$ in animal model (13). For the experiment, the C57BL/ 6 mice were randomly distributed into five groups ( $n=4-12$ per group): healthy (normal mice treated with drinking water ad libitum, oral, $n=8$ ); the DSS/Control (DSS-induced UC group treated with vehicle solution, oral, $n=12$ ); the DSS/ASA (DSS-induced UC group treated with $75 \mathrm{mg} /$ $\mathrm{kg} / \mathrm{d}$ 5-ASA, oral, $n=8$ ); the DSS/TRF (DSS-induced UC group supplemented with $150 \mathrm{mg} / \mathrm{kg} / \mathrm{d}$ TRF, oral, $n=4$ ), the DSS/ $\alpha \mathrm{TP}$ (DSS-induced UC group supplemented with $150 \mathrm{mg} / \mathrm{kg} / \mathrm{d} \alpha \mathrm{TP}$, oral, $n=4)$.

One week prior to the DSS-induced UC, two groups of mice were pre-supplemented with TRF and $\alpha \mathrm{TP}$ respectively for a week (from day 0 to day 7 ), as shown in Fig. 1. In order to achieve higher systemic bioavailability, a self-emulsifying delivery system, which consisted of tween $80(25 \% \mathrm{w} / \mathrm{w})$, span $20(5 \% \mathrm{w} / \mathrm{w})$ and soya oil $(20 \% \mathrm{w} / \mathrm{w})$, was used to prepare TRF and $\alpha \mathrm{TP}(50 \%$ $\mathrm{w} / \mathrm{w})$ in water-dispersible form respectively. 5-ASA was prepared by dissolving $15 \mathrm{mg}$ in $0.1 \mathrm{~mL}$ DMSO and this stock solution was further diluted with $1.9 \mathrm{~mL}$ of autoclaved water. One week later, all groups, except the healthy group, were administered a course of $5 \%$ DSS to induce the UC for 5 consecutive days (from day 8 to day 12). During DSS administration, DSS/Control animal group received vehicle solution, DSS/ASA animal group received $75 \mathrm{mg} / \mathrm{kg} / \mathrm{d} 5$-ASA, DSS/TRF group and DSS/ $\alpha \mathrm{TP}$ group animals received $150 \mathrm{mg} / \mathrm{kg} / \mathrm{d}$ TRF and $\alpha \mathrm{TP}$ respectively. After the cessation of DSS administration, all the groups continue to receive the daily treatments for one more week (from day 13 to day 19). During the duration of the experiment, a disease activity index (DAI) score was used to evaluate the clinical progression of UC on day $8,10,12,15$, and 17 . After the treatment duration (day 20), the mice were fasted $24 \mathrm{~h}$ before they were sacrificed by carbon dioxide asphyxiation. Blood 


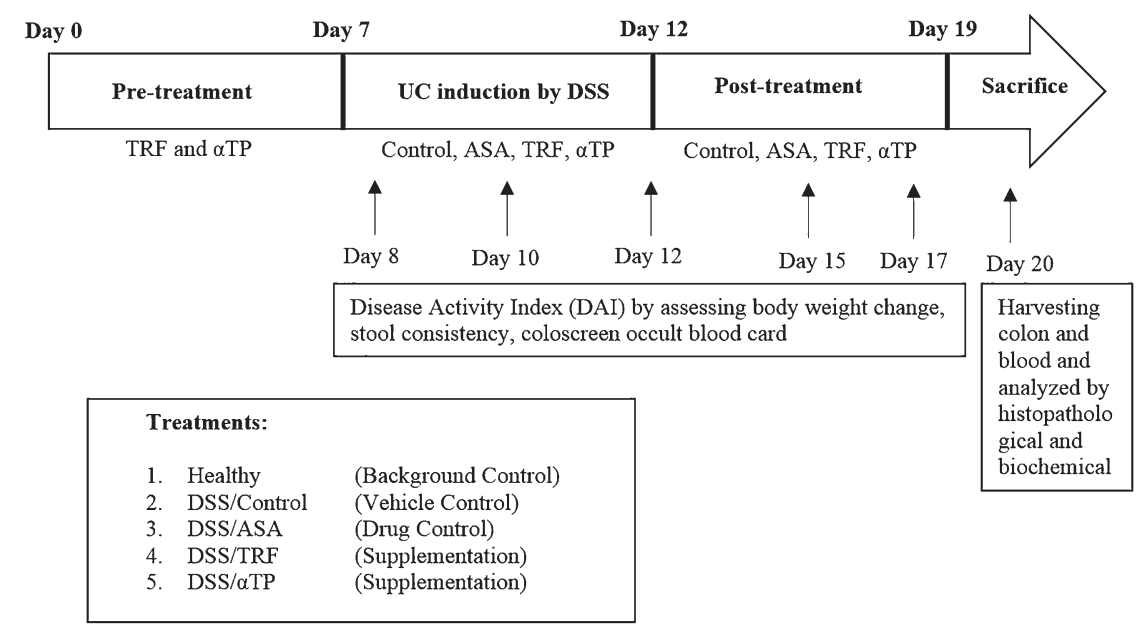

Fig. 1. Schematic of the experimental design. DSS-induced mice were treated with 5 -ASA (75 mg/kg/d), TRF (150 mg/ $\mathrm{kg} / \mathrm{d}), \alpha \mathrm{TP}(150 \mathrm{mg} / \mathrm{kg} / \mathrm{d})$, as described in "Materials and Methods." 5-ASA was used as a standard reference substance.

samples were collected by cardiac puncture and centrifuged at 3,000 $\times g$ for 15 min and plasma were collected and stored at $-80^{\circ} \mathrm{C}$ until further analyses. Whole colons were harvested and the length of colons from the base of cecum to the rectum was measured. Two centimeters of proximal colons were cut and weighed, and then stored at $-80^{\circ} \mathrm{C}$ until they were needed for biochemical analyses. The remaining colon segment was cleaned with ice-cold saline and it was then fixed in 10\% buffered formalin overnight for subsequent histopathological analysis. Spleens were also harvested, washed with ice-cold saline, blotted and weighed.

Disease activity index (DAI). Body weight, stool consistency and presence of faecal blood were measured and assessed every 2 to $3 \mathrm{~d}$ after DSS induction started. These are three key components in computing DAI that quantify the severity of $\mathrm{UC}$ as described in literature (14). For body weight change, score 0 was given to animals with $\leq 1 \%$ weight change; score 1 for 1 to $<5 \%$ weight change; score 2 for 5 to $<10 \%$ weight change; score 3 for 10 to $<15 \%$ weight change; and score 4 for $\geq 15 \%$ weight change. For stool consistency, score 0 was given to normal, dry and solid stool; score 2 for loose stool; and score 4 for diarrheic excrement. For faecal blood which tested using Hemoccult Sensa slides (Beckman Coulter Inc., Brea, CA), score 0 was given to stool with no blood; score 2 for stool with blood; and score 4 for visible gross bleeding. DAI was then computed by taking the average of these three indices.

Histopathological inflammatory scoring. The formalin-fixed colons were rolled into a flat and compact spiral and embedded in wax paraffin. Histological slides were sectioned at $5 \mu \mathrm{m}$ and stained with haematoxylin and eosin (H\&E). Two slides from each group were selected and tissue damage was assessed in a blinded manner by a certified pathologist from histological lab of A*STAR, Singapore. A histopathological score that qualitatively characterize the severity of epithelial damage in accordance to a system described previously was rated for each group (15). The inflammation scoring was deter- mined based on four aspects: (a) inflammation severity (0, normal; 1 , mild inflammation; 2 , moderate inflammation; 3, severe inflammation), (b) ulceration (0, absent; 1 , present), (c) area of inflammation $(0,0 \% ; 1$, $1-25 \% ; 2,26-50 \% ; 3,51-75 \% ; 4,76-100 \%)$ and (d) hyperplasia and dysplasia ( 0 , normal; 1 , mild hyperplasia; 2, low grade dysplasia; 3, high-grade dysplasia).

Detection and quantification of inflammatory and oxidative biomarkers. Proximal colons were thawed on ice and then mechanically homogenized in $1 \mathrm{~mL}$ ice-cold radioimmunoprecipitation assay (RIPA) lysis buffer containing protease and phosphatase inhibitors (Roche Diagnostics GmbH, Mannheim, Germany). The homogenized lysates were centrifuged at $15,000 \times g$ at $4^{\circ} \mathrm{C}$ for $15 \mathrm{~min}$, and the supernatants were extracted. Inflammatory biomarkers: TNF- $\alpha$, IL-6, IL-17, MPO and COX-2 were quantified using ELISA kits (R\&D Systems, Minneapolis, MN). Oxidative biomarker: malondialdehyde (MDA), the end-product of lipid peroxidation was also quantified by ELISA kits (Cell Biolabs Inc., San Diego, $\mathrm{CA}$ ). The nitrite accumulation in the colonic tissues was measured using Griess reagent kit (Molecular Probes Inc., Eugene, OR). Phospho-NF- $\kappa$ B p65 (Ser536) levels were determined using PathScan ${ }^{\circledR}$ ELISA kit (Cell Signalling Technology, Danvers, MA). All procedures were performed as per manufacturers' instructions. The colorimetric reactions were measured with an automated ELISA microplate reader (EnSpire ${ }^{\circledR}$ Multimode Reader; Perkin Elmer, Waltham, MA). Values were expressed as relative fold-change that normalized to that of healthy group.

Extraction of TRF and $\alpha$ TP from colonic tissues and plasma. Colon lysate $(200 \mu \mathrm{L}$ of supernatant) or blood plasma from each mouse were processed via liquid-liquid extraction techniques. In brief, $1 \mathrm{~mL}$ of $0.01 \%$ butylated hydroxytoluene (BHT; Sigma-Aldrich GmBH, Steinhelm, Germany) in ethanol was added to the samples to minimise oxidative degradation of target analyte during processing. Each sample was spiked with $10 \mu \mathrm{L}$ of $0.5 \mathrm{mg} /$ $\mathrm{mL}$ 2,2,5,7,8-pentamethyl-6-chromanol (PMC; Sigma- 
Aldrich, St. Louis, MO) in hexane. PMC was used as an internal control to verify the percentage sample recovery in the extraction process. Two milliliters hexane was added and the mixture was vortex vigorously for $5 \mathrm{~min}$, followed by centrifugation at $1,500 \times g$ for $10 \mathrm{~min}$. The upper hexane phase, which contains hydrophobic vitamin E, was collected. Extraction was repeated twice and all the hexane was pooled into a $10 \mathrm{~mL}$ round bottom flask. The round bottom flasks were then connected to Buchi R-205 Rotavapor (Flawil, Switzerland) to evaporate the solvent. Remaining extract was reconstituted in $300 \mu \mathrm{L}$ hexane and filtered through a $0.2 \mu \mathrm{m}$ filter into a glass vial for chromatographic analysis.

Quantification of TRF and $\alpha$ TP using high performance liquid chromatography (HPLC). Ten microliters of sample was injected by auto-sampler into Agilent 1100 Series HPLC system (Agilent Technologies, Santa Clara, CA). The mobile phase was made up of $97 \%$ hexane : $2.5 \%$ dioxane : $0.5 \%$ isopropanol $(\mathrm{v} / \mathrm{v} / \mathrm{v})$. The chromatographic separation was performed in a LiChrospher ${ }^{\circledR}$ Silica60 analytical column $(250 \times 4 \mathrm{~mm} ; 5 \mu \mathrm{m}$ particle size). Flow rate was set isocratically at $1 \mathrm{~mL} / \mathrm{min}$. The absorbance of TRF and $\alpha \mathrm{TP}$ was monitored by a fluorescence light detector with excitation wavelength set at $295 \mathrm{~nm}$ and emission wavelength at $330 \mathrm{~nm}$. Reference standard curves of each vitamin E homologues were individually established from prepared solutions of pure homologues of known concentrations. The concentration of vitamin $\mathrm{E}$ homologues from the colonic tissues and blood plasma of mice were quantified by area-under-the-curve (AUC) after compensation of the recovery yield, in reference with the standard curves.

Statistical analysis. Statistical analyses were performed using GraphPad Prism version 7.02 (GraphPad Software, San Diego, CA). The one-way analysis of variance (ANOVA) followed by Bonferroni's post hoc test was used to compare the difference between the groups. $p<0.05\left(^{*}\right)$ was considered as statistically significant. Other values were indicated as $p<0.01\left(^{* *}\right), p<0.001$ $(* * *)$ and $p<0.0001\left(^{(* * *)}\right.$. All data were expressed as mean \pm standard deviation (SD) except otherwise stated.

\section{RESULTS}

TRF ameliorates DSS-induced clinical symptoms of UC and disease progression

Disease activity index (DAI)

DAI is a quantitative assessment of acute clinical symptoms that are associated with the severity of UC. The higher the DAI (average score of body weight loss, stool consistency and bleeding), the more severe the disease has progressed. In this study, average DAI scores were only collected after the mice were induced with DSS, which started from day 8, 10 and 12. This assessment continued during the recovery phase at day 15 and 17. As shown in Fig. 2A, DSS-induced mice that were treated with $\alpha \mathrm{TP}$ (DSS/ $\alpha \mathrm{TP})$ showed high DAI scores, indicating no protective effect of $\alpha \mathrm{TP}$ in the UC progression, as compared to DSS/Control. On the other hand, the mice that were supplemented with TRF (DSS/ TRF) alleviated the clinical symptoms significantly in
(A)

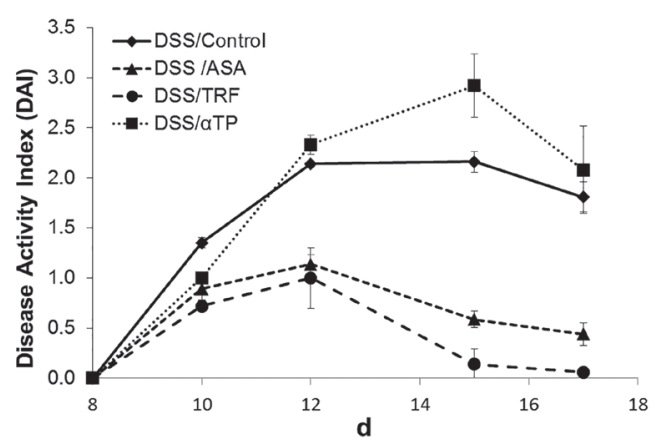

(B)

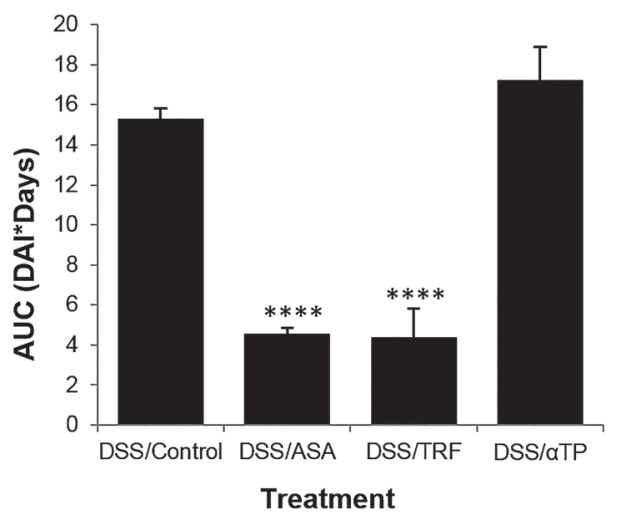

Fig. 2. Effect of oral supplementation of TRF and $\alpha \mathrm{TP}$ on DSS-induced UC in mice. (A) Disease activity index (DAI), an indication of UC severity, of all groups from day 8 to day 17 . DAI was calculated based on number grading given by assessing reduction in body weight, stool quality, and presence of blood in stool. (B) Comparison of the AUC for the change in DAI over time is shown. AUC was calculated using the trapezium rule. The smaller the AUC value, the less severe of the UC condition. Data are expressed as mean \pm standard deviation. ${ }^{* * *} p<0.0001$ vs DSS/Control group, assessed by repeated measures ANOVA.

comparison with DSS/Control on day 15 and day 17 $(p<0.001)$. Similarly, 5-ASA treatment (DSS/ASA) yielded a significantly lower DAI scores compared to DSS/Control $(p<0.001)$. An integrated method was adopted to compare the UC progression over whole treatment period. The AUC is a statistical method that represents the effects of the test substances over the whole trial period of $9 \mathrm{~d}$. The AUC data in Fig. 2A was calculated and presented in Fig. 2B. In this analysis, the smaller the AUC value, the less severe of the UC condition. Both TRF and 5-ASA were effective in ameliorating severity of UC after DSS induction $(p<0.0001)$.

Post-mortem analysis of colon and spleen

In addition to increasing of DAI score, DSS administration also leads to shortened colons and enlargement of spleen in mice, as indicators for the severity of intestinal inflammation, correlate with the pathologic and histological changes and they are consistent markers for colitis (16). Table 1 demonstrates the full colon length, proximal colon weight and spleen weight from all the experimental groups. The colon length of DSS-induced 
Table 1. Measurement of full colon length, proximal colon weight, and spleen weight of all groups at the end of the study.

\begin{tabular}{lccc}
\hline Treatment & Colon length $(\mathrm{cm})$ & Proximal colon weight $(\mathrm{mg})$ & Spleen weight $(\mathrm{mg})$ \\
\hline Healthy & $7.06 \pm 0.68^{* * * *}$ & $83.05 \pm 12.43^{* * * * *}$ & $84.00 \pm 8.74^{* * * *}$ \\
DSS/Control & $4.92 \pm 0.73$ & $141.27 \pm 32.41$ & $143.04 \pm 20.27$ \\
DSS/ASA & $6.36 \pm 0.56^{* * *}$ & $69.84 \pm 17.25^{* * * *}$ & $95.13 \pm 9.89^{* * * *}$ \\
DSS/TRF & $7.13 \pm 0.25^{* * * *}$ & $86.40 \pm 7.36^{*}$ & $109.75 \pm 11.27^{*}$ \\
DSS $/ \alpha$ TP & $5.38 \pm 0.25$ & $120.18 \pm 13.68$ & $121.00 \pm 18.17$ \\
\hline
\end{tabular}

Data are expressed as mean \pm standard deviation. ${ }^{*} p<0.05,{ }^{* * *} p<0.001,{ }^{* * * *} p<0.0001 \mathrm{vs}$ DSS/Control group, assessed by one-way ANOVA, followed by Bonferroni's post hoc test.
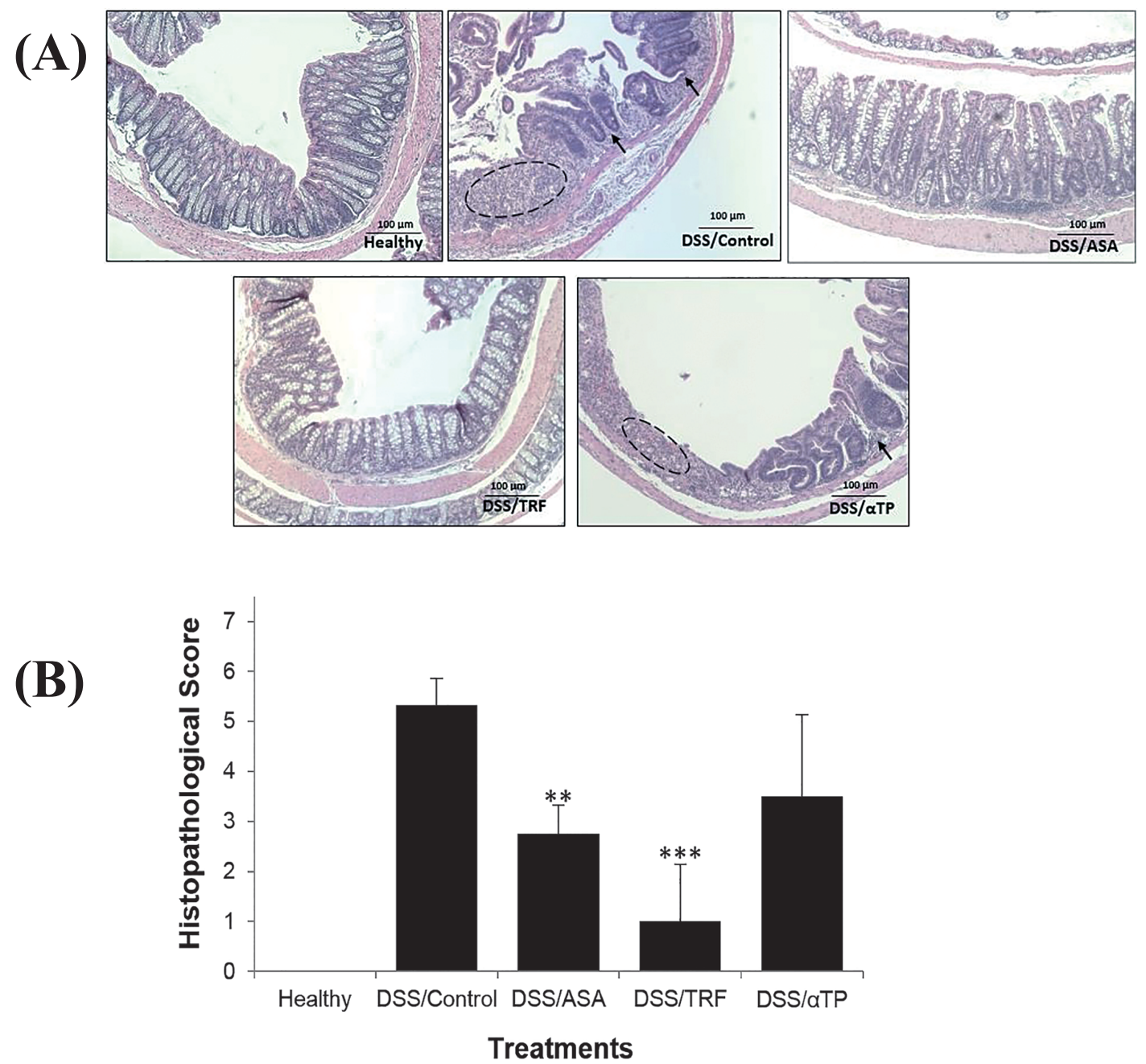

Fig. 3. Histology (haematoxylin \& eosin staining) section of colons obtained from mice. (A) Histopathological analysis (magnification $100 \times$ ) of the colon showing the groups of healthy, DSS/Control, DSS/ASA, DSS/TRF, and DSS/ $\alpha$ TP. Circles with dotted outline show infiltration of inflammatory cells; arrows show disorganization in the architecture of lamina propria. Scale bars are indicated in the lower right corner of each H\&E images. (B) Histopathological score indicated damage in colon microscopic structure, for all groups. The scores were graded in a blinded manner by a certified pathologist based on inflammation severity, ulceration, area of inflammation, and hyperplasia and dysplasia. Data are expressed as mean \pm standard deviation. ${ }^{* *} p<0.01,{ }^{* * *} p<0.001$ vs DSS/Control group, assessed by one-way ANOVA, followed by Bonferroni's post hoc test.

mice treated with vehicle (DSS/Control) was significantly shorter than that of the healthy mice $(4.92 \pm 0.73 \mathrm{~cm}$ vs $7.06 \pm 0.68 \mathrm{~cm}, p<0.0001)$. In comparison to healthy group, DSS/Control colons were heavier in weight

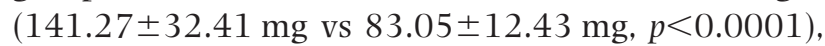
and their spleens were swollen $(143.04 \pm 20.27 \mathrm{mg}$ vs
$84.00 \pm 8.74 \mathrm{mg}, p<0.0001)$ as a response to the local inflammatory reactions. In contrast, the mice supplemented with TRF (DSS/TRF) was able to preserve the colon lengths, maintain colon weights and reduce splenomegaly significantly $(p<0.0001, p<0.05$ and $p<0.05$ respectively). Treatment with 5 -ASA has shown 
(A)

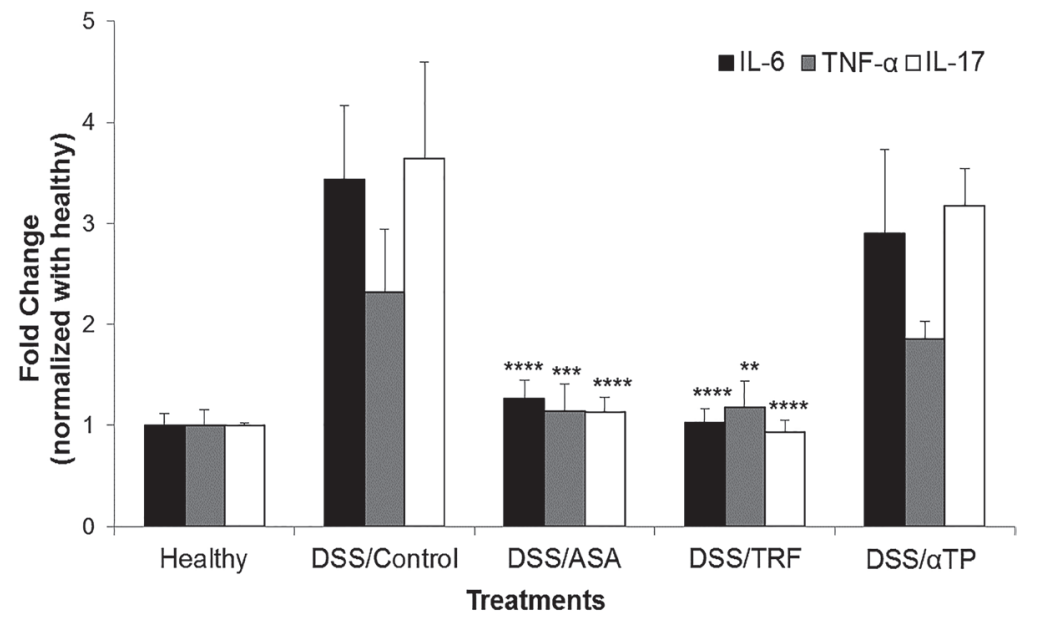

(B)

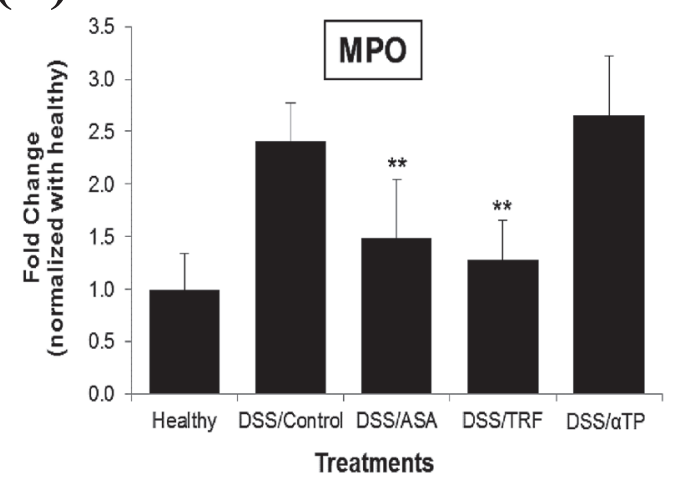

(D)

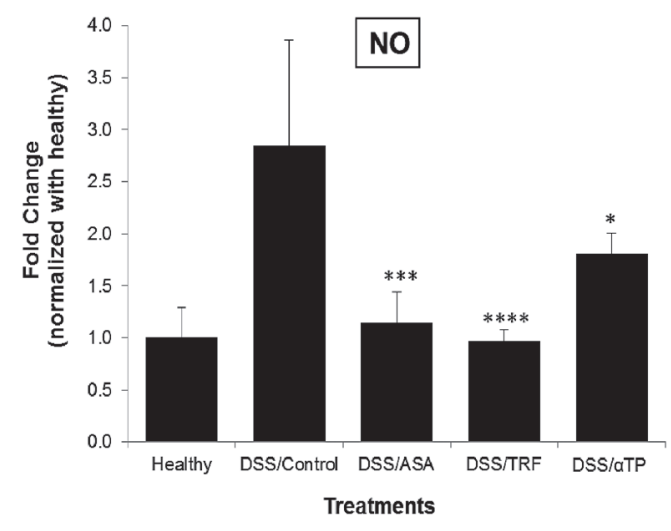

(C)

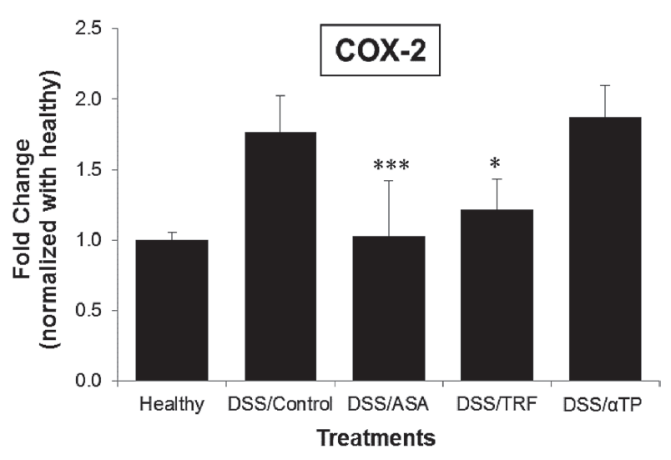

(E)

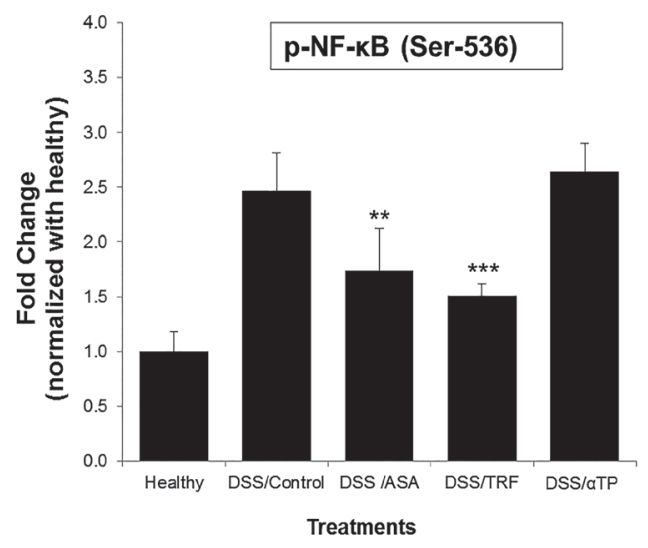

Fig. 4. Anti-inflammatory properties of treatment groups. Levels of (A) proinflammatory cytokines (IL-6, TNF- $\alpha$, IL-17), (B) MPO, (C) COX-2, (D) NO, (E) p-NF- $\kappa \mathrm{B}$ (ser-536) in the colonic tissues of mice that were treated with vehicle, $75 \mathrm{mg} / \mathrm{kg} / \mathrm{d}$ 5 -ASA, $150 \mathrm{mg} / \mathrm{kg} / \mathrm{d}$ TRF or $150 \mathrm{mg} / \mathrm{kg} / \mathrm{d} \alpha \mathrm{TP}$. Data are expressed as mean \pm standard deviation. ${ }^{*} p<0.05,{ }^{* *} p<0.01$, ${ }^{* * *} p<0.001$, and ${ }^{* * * *} p<0.0001$ vs DSS/Control group, assessed by one-way ANOVA, followed by Bonferroni's post hoc test.

similar effectiveness as TRF. However, $\alpha$ TP-treated mice (DSS/ $\alpha \mathrm{TP})$ failed to restore the colon macroscopic observation with parameters comparable to vehicle-treated DSS-induced mice (DSS/Control).

\section{Histopathological observation and scoring}

Figure 3A demonstrates the histopathological examination of DSS-induced vehicle treated mice (DSS/Con- trol) showed destruction of colon epithelial architecture with apparent loss of intestinal crypts, goblet cells and epithelial integrity. Furthermore, infiltration of inflammatory cells into the epithelium was evident in these mice. This is contrast to the normal colonic epithelium of healthy mice. Preventive measure with supplementation of TRF and 5-ASA demonstrated colonic improvement 


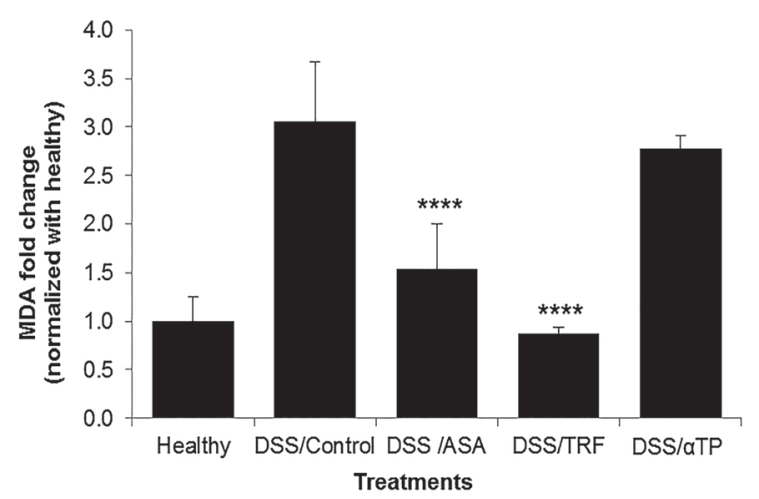

Fig. 5. Levels of malondialdehyde (MDA), a lipid peroxidation biomarker, were assessed by ELISA assay in colonic tissue homogenates of mice receiving either vehicle, $75 \mathrm{mg} / \mathrm{kg} / \mathrm{d} 5$-ASA, $150 \mathrm{mg} / \mathrm{kg} / \mathrm{d}$ TRF or $150 \mathrm{mg} / \mathrm{kg} / \mathrm{d} \alpha \mathrm{TP}$. Data are expressed as mean \pm standard deviation. ${ }^{* * * *} p<0.0001$ vs DSS/Control group, assessed by one-way ANOVA, followed by Bonferroni's post hoc test.

with less inflammatory cells as well as reduced mucosal damage. Treatment of $\alpha \mathrm{TP}$ had persistent infiltration of inflammatory cells together with loss of normal colonic structures which were similar to that of DSS/Control. H\&E-stained tissue slides of the colons were graded and presented in histopathological score by a professional veterinary pathologist in a blinded manner to further confirmed the above microscopic observations based on inflammation severity, ulceration, area of inflammation, hyperplasia and dysplasia. As shown in Fig. 3B, DSS/ Control group had the highest histopathological score (5.33 \pm 0.58$)$ which indicated the severe inflammation of the colon with high grade of dysplasia. It was characterized by epithelial ulceration and erosion which was the worst damage among all groups. After accumulative TRF supplementation for $19 \mathrm{~d}$, there was a significant decrease in scores as compared to with DSS/Control mice $(1.00 \pm 1.15, p<0.001)$. This corresponded to a histologic change that may be barely noticeable in the inflammation severity and ulceration in the TRF-supplemented mice. The same effect was observed in 5-ASA treatment $(2.75 \pm 0.53, p<0.01)$. There was no detectable difference in the histopathological score for the $\alpha \mathrm{TP}$ supplementation group (3.50 \pm 0.41$)$, which indicated the severity of inflammation and ulceration were noticeable but not a prominent feature for the histologic change.

TRF alleviates DSS-induced inflammation

As shown in Fig. 4A, administration of DSS in mice resulted in a 2 to 3.5 -fold increase in IL-6, TNF- $\alpha$ and IL-17 pro-inflammatory cytokine levels in colonic tissues. The activation of these cytokines was accompanied by neutrophil infiltration into the colonic tissues as evidenced by a 2-fold upregulation of MPO level produced by these cells denoting inflammatory activity (Fig. 4B). Our findings showed that supplementation of TRF and 5-ASA significantly attenuated three proinflammatory cytokines, followed by a reduction in MPO
(A)

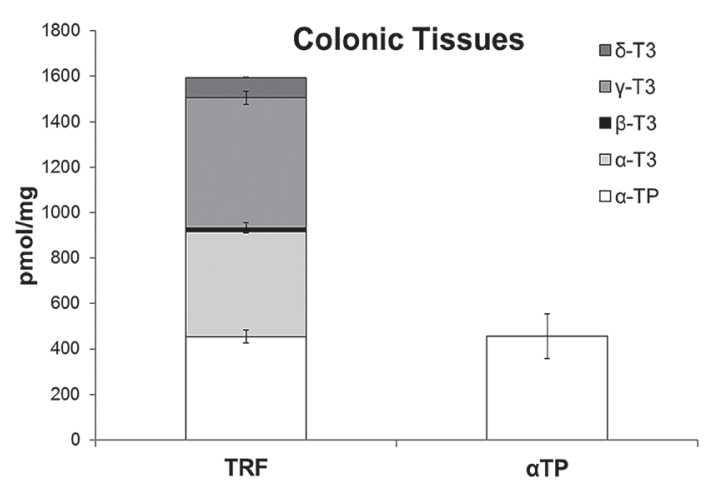

(B)

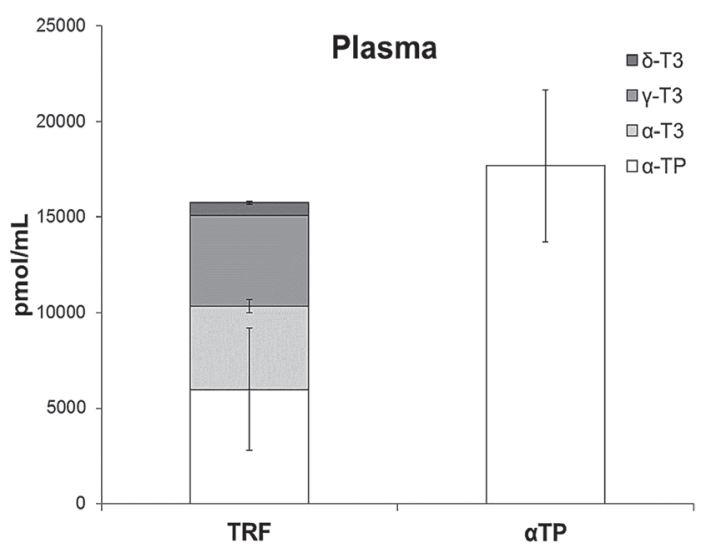

Fig. 6. Vitamin E absorption of TRF and $\alpha \mathrm{TP}$ in (A) colonic tissues, and (B) plasma using high performance liquid chromatography at day 20. Each bar is expressed as mean \pm standard deviation.

level. During immunopathogenesis of IBD, iNOS activation leads to excessive production of NO, subsequently stimulating COX-2 activities. As shown in Fig. 4C and $4 \mathrm{D}, \mathrm{DSS} /$ Control group significantly induced COX-2 and NO levels by 1.8 - and 2.8-fold respectively, whereas the activities were significantly downregulated by TRF and 5-ASA treatments in the colon of the mice. In contrast, with $\alpha \mathrm{TP}$ treatment, MPO and inflammatory cytokines levels still remain similar to that of DSS/Control.

These proinflammatory cytokines mediators are modulated by transcription factors including NF- $\kappa$ B. Phosphorylation of NF- $\kappa \mathrm{B}$ p 65 on serine- 536 has been associated with enhanced NF- $\kappa \mathrm{B}$ activity, which leads to gene transcriptional regulation. As shown in Fig. 4E, TRF and 5-ASA treatments were found to suppress the NF- $\kappa \mathrm{B}$ activity by reducing phospho- 65 levels in the colons of DSS challenged mice ( $p<0.001, p<0.01$ respectively). There were no detectable differences between $\alpha \mathrm{TP}$ and DSS/Control.

\section{TRF alleviates DSS-induced oxidative stress}

The level of MDA, a biomarker for lipid peroxidation was also increased by 3-fold in the colonic tissue (Fig. 5). TRF and 5-ASA treatment significantly reduced levels of MDA by $0.9-$ and 1.5 -fold respectively, but not $\alpha \mathrm{TP}$, as compared to DSS/Control.

TRF shows greater absorption in colonic tissue

The higher potency of TRF in ameliorating the clinical symptoms of DSS-induced colitis including the 
inflammation and oxidative stress may be ascribed to the accelerated cellular uptake by the epithelium colon cells. According to the Fig. 6, absorptions of these two vitamin E forms-TRF and $\alpha$ TP after the oral supplementation were compared in colonic tissues and plasma level. Figure 6A showed that at the same dosage, TRF yielded at least 4-fold greater absorption into the colonic tissues as compared to $\alpha \mathrm{TP}(p<0.05)$. The level of $\alpha \mathrm{TP}$ in both groups were similar (400 pmol/mg protein) but the four isomers of T3 in TRF caused a profound increase in the total vitamin E concentration in colon. In contrast, the concentration of TRF in plasma level after oral supplementation was not significant as compared to the $\alpha \mathrm{TP}$ supplementation which showed the similar amount of concentration (Fig. 6B).

\section{DISCUSSION}

As with other autoimmune diseases, UC has no cure so patients often resort to palliative treatments when relapse happens. The occurrence and duration of relapse depends on myriad of factors and prediction is often challenging. Supplementation with antioxidant and anti-inflammatory agent is a possible way of UC disease management. Preparative nourishment to the local tissue can prevent local immune responses from flaring up, so as to prevent the initiation of relapse and to alleviate the symptoms when relapses happen. There are numbers of research work on IBD animal models using $\alpha \mathrm{TP}$ to treat UC have been published (17-19). Apart from the use of TP in IBD, there had been reports indicating the use of tocotrienol on gastrointestinal related disorders, including stress-induced gastric lesions and ulcers (20). In another study, T3 has demonstrated antifibrogenic effects in human intestinal fibroblasts in vitro (21). In this in vitro study, T3 was shown to reduce cell proliferation, promote cell death and decrease extracellular matrix production and thus it could be beneficial to treat bowel fibrosis. However, the study was not targeting the treatment of IBD but focused mainly on treating or preventing bowel fibrosis that develop during Crohn's disease (CD) progression. Our study is the first to investigate the anti-inflammatory potential of TRF and compare the effects against $\alpha \mathrm{TP}$ as well as the standard treatment option (5-ASA) using a DSS-induced UC murine model.

A hallmark of IBD is an increase in the level of proinflammatory cytokines. A large number of proinflammatory cytokines such as IL-1 $\beta$, IL-6, IL-8, IL-12, IL-17 or TNF- $\alpha$ are known to be upregulated during IBD and they are associated with the initiation and progression of UC and CD (22). The activities of NO and COX-2 has also been shown to contribute to this disease $(23,24)$. iNOS activation lead to excessive production of NO, which causes the stimulation of COX-2 activity. The upregulated COX-2 is responsible for the overproduction of prostaglandin E2 (PGE2), which is a proinflammatory eicosanoid causing pain and fever. In our DSS-induced UC model, we demonstrated elevations in proinflammatory cytokines namely IL-6, TNF- $\alpha$ and IL-17. Such increase in inflammatory mediators was supported with microscopic evidences whereby massive inflammatory immune cells, responsible for the release of cytokines and reactive species, infiltrated into the colonic tissues causing marked mucosal distortion and tissue damage. Furthermore, activated macrophages and neutrophils generate excess amount of highly toxic ROS and RNS, which exceeds the limited intestinal antioxidant defense system, thereby contributing to intestinal oxidative injury in patients (25). Increased in oxidative stress marker such as MDA (reactive aldehydes from lipid peroxidation) levels were also noted in our model.

The present study demonstrates that TRF is an effective inhibitor of proinflammatory cytokines, COX-2, NO as well as MDA production in DSS-induced UC. Mice treated with TRF clearly demonstrated a lower DAI index with reduced infiltration of immune cells and restored mucosal epithelium. TRF treatment alleviated UC symptoms similar to 5-ASA which is the standardof-care medication. Possible mechanism could be linked to the ability of TRF to suppress the activation of NF- $\kappa \mathrm{B}$, which is a crucial transcription factor involved in mediating inflammation pathways. In IBD, NF- $\kappa \mathrm{B}$ has been identified as one of the key regulators resulting in hyperactivation of effector immune cells, which produce high levels of key inflammatory mediators such as COX-2, iNOS, TNF- $\alpha$, IL- 6 and IL- 8 , resulting in colonic tissue damage (26). NF- $\kappa \mathrm{B}$ levels are elevated in IBD patients, in particular the p65 subunit was shown to be highly activated in epithelial cells and macrophages from patients with active UC and CD (27). In fact, number of standard interventions in IBD such as corticosteroids, 5-ASA, sulfasalazine and anti-TNF- $\alpha$ antibodies are known to mediate their anti-inflammatory effects via the inhibition of NF- $\kappa$ B activity $(28-30)$. Our data showed that TRF was able to suppress NF- $\kappa \mathrm{B}$ activity, similar to the effect of 5-ASA. In addition, TRF inhibited expression of IL-17 and TNF- $\alpha$ which are known as inducers of NF- $\kappa \mathrm{B}$ activity (31). These findings provide evidence that TRF possesses anti-inflammatory and antioxidative properties possibly by targeting NF- $\kappa \mathrm{B}$ activity, thereby inhibiting the induction of COX-2 expression, proinflammatory cytokines syntheses as well as NO.

While $\alpha \mathrm{TP}$ and its derivatives are the most common vitamin E used in dietary supplementation, studies showed that T3 may have greater antioxidant and antiinflammatory activities compared to $\alpha \mathrm{TP}$ (32). Research found that $\mathrm{T} 3$ is 40 to 60 times more powerful in antioxidation properties than TP in cells (10). The higher antioxidant potency of $\mathrm{T} 3$ was due to the combined effects of three properties: (i) tocotrienol has a higher recycling efficiency from its chromanoxyl radicals than tocopherol, (ii) tocotrienol is significantly less associated in clusters and is more uniformly distributed in membrane bilayers than tocopherol, (iii) tocotrienol has a strong disordering effect on membrane lipids which makes interaction of the chromanols with lipid radicals more efficient (32). Another study demonstrated the ability of TRF to inhibit NF- $\kappa \mathrm{B}$ activation and suppressed the key inflammatory mediators in mouse peritoneal macrophages and was proven to be more potent than $\alpha \mathrm{TP}$ 
and $\alpha \mathrm{TP}$ acetate (11). An in vitro study showed that T3 has a great anti-inflammatory activity than $\alpha \mathrm{TP}$ as measured by lipopolysaccharide-induced production of IL-6, PGE-2 and COX-2 (33).

In this study, we had similar findings indicating TRF was more superior compared to $\alpha$ TP. DSS induced mice treated with TRF showed marked improvement in UC symptoms with significant reduction of inflammatory mediators and cytokines, e.g. IL-6, TNF- $\alpha$, IL-17, COX2, NO and oxidative stress marker, e.g. MDA, compared to $\alpha$ TP treatment. NF- $\kappa$ B activity was reduced significantly with TRF supplementation but not with $\alpha \mathrm{TP}$. These superior protective effects may be ascribed mainly to structural differences of T3 and TP. T3 contains an unsaturated phytyl chain and the presence of these three double bonds might result in less planar molecular conformation that facilities passage of T3 into liposomal membranes more readily than TP. This may lead to higher cellular uptake of $\mathrm{T} 3$ and thus contribute to greater biopotency $(34,35)$. In our study, we analyzed vitamin E levels present in the colons and plasma of mice supplemented with TRF and those supplemented with $\alpha$ TP. It was observed that $\alpha$ TP levels present in the colon after $7 \mathrm{~d}$ were 3.5-fold lower compared to TRF, at the same supplementation dosage. This is in agreement with earlier studies that showed higher cellular uptake of T3 as compared to TP in hepatocytes, human T-leukemia cell, primary cortical neuron cells and human lung carcinoma cells. This has been attributed to the higher intermembrane mobility of the tocotrienol (35-38). However, TRF and $\alpha \mathrm{TP}$ showed similar pharmacologic effect in plasma. These finding has speculated the accumulation of TRF in the colon cells - not the plasma concentration-is the key determinant of biological effectiveness of TRF in this DSS-induced UC murine model.

It is noteworthy that the dosage used in this study (150 mg/kg/d of TRF) demonstrate restoration of normal colons in these mice. Safety dosage of T3 supplementation has been studies in different studies. In one study, TRF administrated to young Sprague-Dawley rats (weight 150-180 g) and albino mice (weighing $15-20 \mathrm{~g}$ ) at a dose of up to $2,500 \mathrm{mg} / \mathrm{kg}$ for $30 \mathrm{~d}$ showed adverse effects in physical manifestations or behavioural changes (39). In another study, a 52-wk toxicity study performed in rats showed no-observedadverse-effects levels (NOAEL) were at $303 \mathrm{mg} / \mathrm{kg} / \mathrm{d}$ for males and $472 \mathrm{mg} / \mathrm{kg} / \mathrm{d}$ for females (40). The Antioxidant Panel of the Food and Nutrition Board at the Institute of Medicine of the US National Academy of Sciences reported that vitamin $\mathrm{E}$ is safe for chronic use by the general population at doses up to $1,000 \mathrm{mg} / \mathrm{d}$ in adults (41). In our study, the orally administered dose of TRF in mice $(150 \mathrm{mg} / \mathrm{kg} / \mathrm{d})$ correspond to a human equivalent dose of $730 \mathrm{mg} / \mathrm{d}$ according to body surface area comparison between mice weighing $20 \mathrm{~g}$ and adult humans weighing $60 \mathrm{~kg}$. This extrapolated non-toxic dose of $730 \mathrm{mg} / \mathrm{d}$ is lower than the upper tolerable limit of $1,000 \mathrm{mg} / \mathrm{d}$. Therefore, the dose of $150 \mathrm{mg} / \mathrm{kg}$ in mice that was effective in alleviating the symptoms of $\mathrm{UC}$ is safe when extrapolated to humans. As standard treatments including 5-ASA and corticosteroids may lead to undesirable adverse effects, naturally occurring TRF may provide a safer alternative treatment option of UC with comparable efficacy to the standard treatments and possibly fewer side effects.

In the present study, TRF supplementation effectively suppressed inflammation and oxidative stress-associated UC. Our findings showed that TRF, but not $\alpha \mathrm{TP}$, attenuated colonic inflammation, reduced oxidative stress as well as inhibited NF- $\kappa \mathrm{B}$ activity, resulting in significant improvement in clinical outcomes. These improvement in clinical sign and symptoms was similar to that of 5-ASA treatment. Taken together, dietary supplementation of TRF may envisage protection against inflammation of gastrointestinal tract. Clinical study is warranted to evaluate the effectiveness of TRF in UC patients.

\section{Disclosure of State of COI}

Tzuen Yih Saw, Najib Abdul Malik, Kee Pah Lim, Cheryl Wei Ling Teo, Chee Wai Fong, Jordan Petkov and Wei Ney Yap work for Davos Life Science (a subsidiary of KLK Oleo), a manufacturer of Tocotrienol. The rest of authors declare that they have no competing interests.

\section{Acknowledgments}

This work was supported financially by a research grant from KL-Kepong Oleomas Sdn Bhd (KLK Oleo) to Davos Life Science Pte. Ltd. The authors would like to thank Ms. Suet Hoay Lee for helping with the animal experimentation and Ms. Wee Ting Tan for the HPLC technical support. We also appreciate the help from Dr. Alvin Loo for input in manuscript design and writing.

\section{REFERENCES}

1) Papadakis KA, Targan SR. 2000. Role of cytokines in the pathogenesis of inflammatory bowel disease. Annu Rev Med 51: 289-298.

2) Gohil K, Carramusa B. 2014. Ulcerative colitis and Crohn's disease. Pharm Ther 39: 576-577.

3) Strober W, Fuss I, Mannon P. 2007. The fundamental basis of inflammatory bowel disease. J Clin Investig 117 : 514-521.

4) Abraham C, Cho JH. 2009. Inflammatory bowel disease. N Engl J Med 361: 2066-2078.

5) Triantafillidis JK, Merikas E, Georgopoulos F. 2011. Current and emerging drugs for the treatment of inflammatory bowel disease. Drug Des Dev Ther 5: 185-210.

6) Richter JM, Kushkuley S, Barrett JA, Oster G. 2012. Treatment of new-onset ulcerative colitis and ulcerative proctitis: a retrospective study. Aliment Pharmacol Ther 36: $248-256$.

7) Baert F, Vermeire S, Noman M, Van Assche G, D’Haens G, Rutgeerts P. 2004. Management of ulcerative colitis and Crohn's disease. Acta Clin Belg 59: 304-314.

8) Kamm MA. 2002. Review article: Maintenance of remission in ulcerative colitis. Aliment Pharmacol Ther 16(Suppl 4): 21-24.

9) Xu CT, Meng SY, Pan BR. 2004. Drug therapy for ulcerative colitis. World J Gastroenterol 10: 2311-2317.

10) Serbinova E, Kagan V, Han D, Packer L. 1991. Free radical recycling and intramembrane mobility in the antioxidant properties of alpha-tocopherol and alpha-toco- 
trienol. Free Radic Biol Med 10: 263-275.

11) Ng LT, Ko HJ. 2012. Comparative effects of tocotrienolrich fraction, alpha-tocopherol and alpha-tocopheryl acetate on inflammatory mediators and nuclear factor kappa B expression in mouse peritoneal macrophages. Food Chem 134: 920-925.

12) Wu SJ, Liu PL, Ng LT. 2008. Tocotrienol-rich fraction of palm oil exhibits anti-inflammatory property by suppressing the expression of inflammatory mediators in human monocytic cells. Mol Nutr Food Res 52: 921-929.

13) Chassaing B, Aitken JD, Malleshappa M, Vijay-Kumar M. 2014. Dextran sulfate sodium (DSS)-induced colitis in mice. Curr Protoc Immunol 104: Unit 15.25.

14) Kim HS, Berstad A. 1992. Experimental colitis in animal models. Scand J Gastroenterol 27: 529-537.

15) Mahler M, Bristol IJ, Leiter EH, Workman AE, Birkenmeier EH, Elson CO, Sundberg JP. 1998. Differential susceptibility of inbred mouse strains to dextran sulfate sodium-induced colitis. Am J Physiol 274: G544-551.

16) Okayasu I, Hatakeyama S, Yamda M, Ohkusa T, Inagaki Y, Nakaya R. 1990. A novel method in the induction of reliable experimental acute and chronic ulceratives colitis in mice. Gastroenterology 98(3): 694-702.

17) Tahan G, Aytac E, Aytekin H, Gunduz F, Dogusoy G, Aydin S, Tahan V, Uzun H. 2011. Vitamin E has a dual effect of anti-inflammatory and antioxidant activities in acetic acid-induced ulcerative colitis in rats. Can J Surg 54: 333-338.

18) Sato K, Kanazawa A, Ota N, Nakamura T, Fujimoto K. 1998. Dietary supplementation of catechins and alphatocopherol accelerates the healing of trinitrobenzene sulfonic acid-induced ulcerative colitis in rats. J Nutr Sci Vitaminol 44: 769-778.

19) Mirbagheri SA, Nezami BG, Assa S, Hajimahmoodi M. 2008. Rectal administration of d-alpha tocopherol for active ulcerative colitis: A preliminary report. World J Gastroenterol 14: 5990-5995.

20) Nur Azlina MF, Kamisah Y, Chua KH, Qodriyah HM. 2013. Tocotrienol attenuates stress-induced gastric lesions via activation of prostaglandin and upregulation of COX-1 mRNA. Evid Based Complement Alternat Med 2013: 804796.

21) Luna J, Masamunt MC, Rickmann M, Mora R, Espana C, Delgado S, Llach J, Vaquero E, Sans M. 2011. Tocotrienols have potent antifibrogenic effects in human intestinal fibroblasts. Inflamm Bowel Dis 17: 732-741.

22) Bamias G, Kaltsa G, Ladas SD. 2011. Cytokines in the pathogenesis of ulcerative colitis. Discov Med 11: 459-467.

23) Shah V, Lyford G, Gores G, Farrugia G. 2004. Nitric oxide in gastrointestinal health and disease. Gastroenterology 126: 903-913.

24) Wang D, DuBois RN. 2010. The role of COX-2 in intestinal inflammation and colorectal cancer. Oncogene 29: 781.

25) Keshavarzian A, Banan A, Farhadi A, Komanduri S, Mutlu E, Zhang Y, Fields JZ. 2003. Increases in free radicals and cytoskeletal protein oxidation and nitration in the colon of patients with inflammatory bowel disease. Gut 52: 720-728.

26) Neurath M, Becker C, Barbulescu K. 1998. Role of $\mathrm{NF}-\kappa \mathrm{B}$ in immune and inflammatory responses in the gut. Gut 43: 856-860.

27) Neurath MF, Fuss I, Schurmann G, Pettersson S, Arnold K, Muller-Lobeck H, Strober W, Herfarth C, Buschenfelde KH. 1998. Cytokine gene transcription by NF-kappa B family members in patients with inflammatory bowel disease. Ann NY Acad Sci 859: 149-159.

28) Ardite E, Panes J, Miranda M, Salas A, Elizalde JI, Sans M, Arce Y, Bordas JM, Fernandez-Checa JC, Pique JM. 1998. Effects of steroid treatment on activation of nuclear factor kappaB in patients with inflammatory bowel disease. Br J Pharmacol 124: 431-433.

29) Gisbert JP, Gomollón F, Maté J, Pajares JM. 2002. Role of 5-aminosalicylic acid (5-asa) in treatment of inflammatory bowel disease: a systemic review. Dig Dis Sci 47: 471-488.

30) Weber CK, Liptay S, Wirth T, Adler G, Schmid RM. 2000. Suppression of NF-kappaB activity by sulfasalazine is mediated by direct inhibition of IkappaB kinases alpha and beta. Gastroenterology 119: 1209-1218.

31) Pahl HL. 1999. Activators and target genes of Rel/ NF- $\kappa$ B transcription factors. Oncogene 18: 6853.

32) Aggarwal BB, Sundaram C, Prasad S, Kannappan R. 2010. Tocotrienols, the vitamin E of the 21st century: its potential against cancer and other chronic diseases. Biochem Pharmacol 80: 1613-1631.

33) Yam M-L, Hafid SRA, Cheng H-M, Nesaretnam K. 2009. Tocotrienols suppress proinflammatory markers and cyclooxygenase-2 expression in RAW264.7 macrophages. Lipids 44: 787-797.

34) Yoshida Y, Saito Y, Jones LS, Shigeri Y. 2007. Chemical reactivities and physical effects in comparison between tocopherols and tocotrienols: physiological significance and prospects as antioxidants. J Biosci Bioeng 104: 439-445.

35) Saito Y, Yoshida Y, Nishio K, Hayakawa M, Niki E. 2004. Characterization of cellular uptake and distribution of vitamin E. Ann NY Acad Sci 1031: 368-375.

36) Sontag TJ, Parker RS. 2007. Influence of major structural features of tocopherols and tocotrienols on their omega-oxidation by tocopherol-omega-hydroxylase. J Lipid Res 48(5): 1090-1098.

37) Saito Y, Nishio K, Akawaza YO, Yamanaka K, Miyama A, Yoshida Y, Noguchi N, Niki E. 2010. Cytoprotective effects of vitamin $\mathrm{E}$ homologues against glutamateinduced cell death in immature primary cortical neuron cultures: tocopherols and tocotrienols exert similar effects by antioxidant function. Free Radic Biol Med 49(10): 1542-1549.

38) Nishio K, Horie M, Akazawa Y, Shichiri M, Iwahashi H, Hagihara Y, Yoshida Y, Niki E. 2013. Attenuation of lipopolysaccharide (LPS)-induced cytotoxicity by tocopherols and tocotrienols. Redox Biol 1: 97-103.

39) Oo S, Chang P, Chan K. 1992. Toxicological and pharmacological studies on palm vitee. Nutr Res 12: S217-S222.

40) Tasaki M, Umemura T, Inoue T, Okamura T, Kuroiwa Y, Ishii Y, Maeda M, Hirose M, Nishikawa A. 2008. Induction of characteristic hepatocyte proliferative lesion with dietary exposure of Wistar Hannover rats to tocotrienol for 1 year. Toxicology 250: 143-150.

41) Monsen, Elaine E. 2000. Dietary reference intakes for the antioxidant nutrients: Vitamin C, vitamin E, selenium and carotenoids. J Am Diet Assoc 100: 637-640. 